\title{
VARIACIÓN MORFOLÓGICA Y PRODUCTIVA DE TRES ESPECIES DE Lupinus EN UN ENSAYO DE CAMPO
}

\section{MORPHOLOGICAL AND PRODUCTIVE VARIATION OF THREE Lupinus SPECIES IN A FIELD TRIAL}

\section{Oscar G. Vázquez-Cuecuecha ${ }^{1}$, Javier López-Upton ${ }^{1}$, Luz del Carmen Lagunes- Espinoza ${ }^{2}$, Carlos Ramírez-Herrera', Pedro A. López ${ }^{3}$, José L. Martínez-y-Pérez ${ }^{4}$}

\begin{abstract}
'Colegio de Postgraduados, Ciencias Forestales. Montecillo, Estado de México, México. ${ }^{2}$ Colegio de Postgraduados, Campus Tabasco, H. Cárdenas, Tabasco, México. ${ }^{3}$ Colegio de Postgraduados, Campus Puebla, Santiago Momoxpan, Puebla, México. ${ }^{4}$ Universidad Autónoma de Tlaxcala, Centro de Investigación en Genética y Ambiente, San Felipe Ixtacuixtla, Tlaxcala, México.
\end{abstract}

*Autor de correspondencia (jlopezupton@gmail.com)

RESUMEN

Lupinus montanus Kunth (Lm), L. campestris Schltdl. \& Cham. (Lc) y L. exaltatus Zucc. (Le) son especies mexicanas con alto contenido de proteína e importante función ecológica. El análisis de características agronómicas permites definir estrategias para su domesticación. Se estableció para las tres especie un ensayo de campo con plantas producidas a partir de semillas procedentes de diferentes localidades del estado de Puebla, a una densidad de 10,000 plantas ha-1 con el objetivo de evaluar las diferencias entre especies y entre poblaciones en supervivencia y características agronómicas, tales como altura de planta, inflorescencias, vainas, semillas y rendimiento de biomasa. Los resultados mostraron una supervivencia de 29,54 y $61 \%$ para $L m, L c$ y $L e$, respectivamente. La especie que presentaró la mayor producción de vainas por planta (347) fue $L c$ y $L m$ la menor (170). El rendimiento en biomasa fue de $2.12 \mathrm{t} \mathrm{ha}^{-1}$ para $L c, 2.08 \mathrm{tha}^{-1}$ para $L$ e y $2.05 \mathrm{tha}^{-1}$ para $L m$. Se concluye que $L c$ y Le son especies de mejor comportamiento en características agronómicas, lo que las hace promisorias para su aprovechamiento.

Palabras clave: Lupinus, biomasa, semillas, poblaciones, región centro-oriente de Puebla, supervivencia.

\section{SUMMARY}

Lupinus montanus Kunth $(L m)$, L. campestris Schltdl. \& Cham. (Lc) and L. exaltatus Zucc. (Le) are Mexican species with high protein content and important ecological function. The analysis of agronomic characteristics will allow to define strategies for their domestication. A field trial was established for the three species with plants produced from seeds from different locations of the state of Puebla at a density of 10,000 plants ha ${ }^{-1}$ with the objective of assessing differences between species and populations in survival and agronomic characteristics such as plant height, inflorescences, pods, seeds and biomass yield. Results showed a survival of 29,54 and $61 \%$ for $L m, L c$ and $L e$, respectively. The species that presented the highest production of pods per plant (347) was $L c$ presented and $L m$ the lowest one (170). The biomass yield was $2.12 \mathrm{t} \mathrm{ha}^{-1}$ for $L c, 2.08 \mathrm{t} \mathrm{ha}^{-1}$ for $L e$, and $2.05 \mathrm{t} \mathrm{ha}^{-1}$ for $L m$. It is concluded that $L c$ and $L e$ are the species with the best agronomic characteristics, which makes them promising for their use.

Index words: Lupinus, biomass, seeds, populations, central-eastern Puebla, survival.

\section{INTRODUCCIÓN}

Se han reportado más de 200 especies de Lupinus en el mundo, de las que alrededor del $90 \%$ se originaron en el norte y sur de América, se distribuyen desde Alaska hasta Argentina y Chile. El resto de ellas está en la región Mediterránea y en el norte de África (Walker y Luckett, 2011). La mayoría de las especies domesticadas y económicamente importantes se aprovechan en Europa como fuente de proteína y aceite (Annicchiarico et al., 2010; Walker y Luckett, 2011). En las especies domesticadas se han seleccionado y mejorado características morfológicas de vaina y semilla, resistencia a enfermedades, así como adaptación a condiciones climáticas que limitan su producción y supervivencia (Chen et al., 2011; LagunesEspinoza et al., 2000; López-Bellido et al., 1994).

En México existen especies no domesticadas de Lupinus, entre ellas L. montanus Kunth, L. campestris Cham. \& Schltdl. y L. exaltatus Zucc., que se caracterizan por tener altas concentraciones de proteína y minerales, potencialmente útiles para la industria alimenticia (Lagunes-Espinoza et al., 2000; 2012; 2013; Pablo-Pérez et al., 2013); sin embargo, los Lupinus presentan alcaloides quinolizidínicos generados por la planta para el combate de herbívoros y microorganismos (Wink, 1992), los que tendrían que eliminarse previo tratamiento antes del consumo y utilizarse como subproducto. En México, se han identificado alcaloides en L. montanus, L. aschenbornii Schauer y L. stipulatus J. Agardh, que han sido probados por su actividad insecticida (Bermúdez-Torres et al., 2009).

Los estudios sobre las especies de Lupinus en México se han enfocado en la caracterización fenotípica con el objetivo de facilitar su manejo y domesticación con fines de reforestación, de restauración ecológica, para el manejo de germoplasma ante el cambio climático, por su 
potencial uso alimenticio e industrial para composición química de algunas especies (Hernández et al., 2008; Lara-Cabrera et al., 2009; Pablo-Pérez et al., 2015; RuizLópez et al., 2000; Soto-Correa et al., 2012). No obstante, para su conservación y uso adecuados como cultivo es indispensable evaluarlos para determinar el potencial de crecimiento y adaptación de las especies y sus procedencias en diferentes sitios e incluso fuera de su hábitat. A través de ensayos en campo se puede estimar e identificar la supervivencia y rendimiento de variables de interés bajo condiciones ambientales diversas (LópezUpton et al., 2004; Soto-Correa et al., 2012). Por lo tanto, el objetivo del estudio fue evaluar las diferencias en supervivencia, crecimiento y producción de biomasa y semilla entre especies y poblaciones de $L$. montanus, $L$. campestris y $L$. exaltatus a través de un ensayo en campo.

\section{MATERIALES Y MÉTODOS}

\section{Recolecta de germoplasma y tratamiento de la semilla}

Las vainas de 30 plantas se cosecharon por población de cada especie (Cuadro 1), con las que se formó una muestra masal por población-especie. De cada población, 200 semillas se desinfestaron con hipoclorito de sodio $0.35 \%$ por 5 min (Vega et al., 1996) y se escarificaron manualmente con una lija de grano medio para madera.

\section{Siembra y manejo de la planta}

Las semillas se sembraron en tubetes de plástico negro con un sustrato de perlita, turba y corteza de pino en una proporción de 15:15:70 v/v/v. El riego fue aplicado con agua corriente a capacidad de campo cada tercer día, por un periodo de $60 \mathrm{~d}$, hasta que las plantas alcanzaron en promedio $25 \mathrm{~cm}$ de altura.

La plantación se realizó en el municipio de Tlachichuca, Puebla (19 05' 42.27" latitud N, $97^{\circ} 25^{\prime}$ 10.42" longitud O), a $2654 \mathrm{msnm}$, temperatura media de $13.1^{\circ} \mathrm{C}$, mínima $1{ }^{\circ} \mathrm{C}$ y máxima $19.8^{\circ} \mathrm{C}$, con una precipitación media anual de $564 \mathrm{~mm}$. Las plantas se distribuyeron bajo un diseño de bloques completos al azar con seis repeticiones y seis individuos por unidad experimental.

\section{Variables registradas}

La supervivencia se registró cuando la planta presentó la inflorescencia. Las variables morfológicas evaluadas fueron altura y biomasa por planta, longitud de inflorescencia, además de las características de biomasa y dimensiones de vainas, número de semillas llenas (bien formadas), vanas, dañadas por plagas y óvulos abortados, y el peso de 100 semillas llenas. Los equipos empleados fueron flexómetro Truper ${ }^{\circledR}$, vernier digital, balanza analítica (SMÒ, Chyo Balance Corporation, Kyoto, Japón) y balanza electrónica con aproximación a 0.1 g (N08110Ò, Ohaus Corporation, Parsippany, New Jersey, USA). Para determinar la materia seca (biomasa), las plantas se deshidrataron en un horno de secado (Riossa, México) a $65^{\circ} \mathrm{C}$ por $48 \mathrm{~h}$.

\section{Análisis estadístico}

Se realizaron análisis de varianza para determinar diferencias entre las especies y poblaciones, considerando a las especies y poblaciones como efectos fijos y a los bloques como efecto aleatorio. Se obtuvieron los valores ajustados debido al desbalance causado por la mortalidad (Littell et al., 1996). Se utilizó el siguiente modelo anidado mixto:

$$
Y_{i j k l}=\mu+B_{i}+E_{j}+B E_{i j}+P(E)_{j k}+B P(E)_{i j k}+\varepsilon_{i j k l}
$$

donde: $Y_{i j k l}$ es el valor observado en I-ésima planta de la k-ésima población de la j-ésima especie en el i-ésimo bloque, $\mu$ es la media general; $B_{i}$ el efecto del $i$-ésimo bloque, $E_{j}$ el efecto de la $j$-ésima especie, $B E_{i j}$ es el efecto de interacción del i-ésimo bloque con la j-ésima especie, $P(E)_{j k}$ es el efecto de la k-ésima población anidada en la j-ésima especie; $B P(E)_{i j k}$ es el efecto de la interacción del i-ésimo bloque con la k-ésima población anidada en la j-ésima especie y $\varepsilon_{i j k l}$ el error.

Para la variable supervivencia se consideraron porcentajes por parcela, los cuales se transformaron con la función arcoseno (valor)-0.5 (Quinn y Keough, 2002). Se utilizaron los procedimientos PROC MIXED con la opción LSMEANS (SAS Institute, 2004) para obtener los valores de las medias ajustadas debido al desbalance causado por la mortalidad.

\section{RESULTADOS Y DISCUSIÓN}

\section{Supervivencia}

La supervivencia promedio fue de $43.3 \%$, con diferencias significativas entre las especies y entre poblaciones de Lupinus, con intervalos en L. campestris de 36.1 a $66.6 \%$, en $L$. exaltatus de 55.5 a $66.6 \%$ y en $L$. montanus de 16.6 a $38.8 \%$ (Cuadros 2 y 3 ). En experimentos con diferentes especies del género Lupinus se ha determinado que la supervivencia de las plantas depende tanto de su condición genética como de las condiciones ambientales del sitio de prueba (Walker y Luckett, 2011). La mayoría de las poblaciones probadas en este ensayo provinieron de una altitud mayor. Allen et al. (1998) mencionaron que al mover germoplasma hacia lugares de menor altitud las plantas 
Cuadro 1. Poblaciones de Lupinus campestris, L. exaltatus y L. montanus de centro-oriente de Puebla, México probadas en ensayo en campo.

\begin{tabular}{|c|c|c|c|c|c|c|}
\hline Especie & Población & Latitud N & Longitud O & Altitud (msnm) & $\begin{array}{l}\text { Temp. media } \\
\text { anual }\left({ }^{\circ} \mathrm{C}\right)^{+}\end{array}$ & $\begin{array}{c}\text { Precip. media } \\
\text { anual }(\mathrm{mm})^{+}\end{array}$ \\
\hline \multirow{9}{*}{$\begin{array}{l}\text { Lupinus } \\
\text { campestris }\end{array}$} & Poxcoatzingo & 19057'13.0" & $98^{\circ} 00^{\prime} 33.5^{\prime \prime}$ & 2481 & 14.7 & 695 \\
\hline & $\begin{array}{l}\text { Laguna de } \\
\text { Atexca }\end{array}$ & 19057'06.1" & $98^{\circ} 02^{\prime 22.2^{\prime \prime}}$ & 2584 & 14.4 & 677 \\
\hline & Laguna Seca & $18^{\circ} 53^{\prime} 3.05^{\prime \prime}$ & 97079'0.06" & 2669 & 14.1 & 650 \\
\hline & San Isidro & $18^{\circ} 54^{\prime} 33.6^{\prime \prime}$ & $97^{\circ} 18^{\prime} 26.4^{\prime \prime}$ & 2794 & 13.6 & 646 \\
\hline & Barranca Honda & $19^{\circ} 01^{\prime} 46.0^{\prime \prime}$ & $97^{\circ} 22^{\prime} 43.1^{\prime \prime}$ & 2903 & 13.5 & 553 \\
\hline & Tlalmotolo I & 19०34'27.4" & $97^{\circ} 43^{\prime} 38.5^{\prime \prime}$ & 2980 & 12.3 & 667 \\
\hline & Tlalmotolo II & 19034'27.4" & $97^{\circ} 43^{\prime} 37.0^{\prime \prime}$ & 2965 & 12.4 & 655 \\
\hline & Zoapan & $19^{\circ} 04^{\prime} 39.2^{\prime \prime}$ & $97^{\circ} 20^{\prime} 54.0^{\prime \prime}$ & 3064 & 12.7 & 619 \\
\hline & Manuel Ávalos & $19^{\circ} 03^{\prime} 50.4^{\prime \prime}$ & $97^{\circ} 22^{\prime} 59.7^{\prime \prime}$ & 2878 & 13.7 & 527 \\
\hline \multirow{2}{*}{$\begin{array}{l}\text { Lupinus } \\
\text { exaltatus }\end{array}$} & Zoapan & 1904'38.3" & $97^{\circ} 21^{\prime} 57.1^{\prime \prime}$ & 2913 & 13.6 & 539 \\
\hline & San Martín & $19^{\circ} 00^{\prime} 52.4^{\prime \prime}$ & $97^{\circ} 22^{\prime} 07.7^{\prime \prime}$ & 3010 & 12.9 & 595 \\
\hline \multirow{9}{*}{$\begin{array}{l}\text { Lupinus } \\
\text { montanus }\end{array}$} & $\begin{array}{l}\text { Texmalaquilla } \\
3100\end{array}$ & $18^{\circ} 57^{\prime} 21.4$ & $97^{\circ} 17^{\prime} 20.3^{\prime \prime}$ & 3100 & 12.2 & 693 \\
\hline & $\begin{array}{l}\text { Texmalaquilla } \\
3300\end{array}$ & $18^{\circ} 57^{\prime} 59.3^{\prime \prime}$ & $97^{\circ} 17^{\prime} 17.4^{\prime \prime}$ & 3300 & 11 & 840 \\
\hline & $\begin{array}{l}\text { Texmalaquilla } \\
3500\end{array}$ & $18^{\circ} 58^{\prime} 30.0^{\prime \prime}$ & 97017'25.0" & 3500 & 9.8 & 1015 \\
\hline & $\begin{array}{l}\text { Texmalaquilla } \\
3700\end{array}$ & $18^{\circ} 58^{\prime} 56.9^{\prime \prime}$ & $97^{\circ} 17^{\prime} 30.2^{\prime \prime}$ & 3700 & 8.7 & 1169 \\
\hline & $\begin{array}{l}\text { Texmalaquilla } \\
3900\end{array}$ & $18^{\circ} 58^{\prime} 58.0^{\prime \prime}$ & 97017'56.0" & 3900 & 7.6 & 1295 \\
\hline & $\begin{array}{l}\text { Texmalaquilla } \\
4100\end{array}$ & $18^{\circ} 59^{\prime} 49.95^{\prime \prime}$ & $97^{\circ} 17^{\prime} 57.0^{\prime \prime}$ & 4100 & 6.6 & 1403 \\
\hline & Tlanalapan & 19015'31.2" & $97^{\circ} 16^{\prime} 09.7^{\prime \prime}$ & 3045 & 12.9 & 662 \\
\hline & San Joaquín & 1907'09.8" & $97^{\circ} 18^{\prime} 25.4^{\prime \prime}$ & 3112 & 12.3 & 683 \\
\hline & Zoapan & $19^{\circ} 04^{\prime} 18.1^{\prime \prime}$ & $97^{\circ} 19^{\prime} 06.7^{\prime \prime}$ & 3358 & 10.7 & 875 \\
\hline
\end{tabular}

${ }^{\dagger}$ Temperatura media anual y precipitación media anual con ANUSPLIN, software para modelar datos en superficies geográficas considerando la latitud (Sáenz-Romero et al. 2010).

son sometidas a un diferencial de estrés hídrico derivado de una tasa mayor de evapotranspiración en los lugares más calurosos (en sitios de menor altitud). Lo anterior pudiera ser un factor determinante para $L$. montanus, que crece a mayor altitud y mayor precipitación, dado que sus poblaciones presentaron valores menores de supervivencia, lo cual puede deberse a la falta de adaptación al nuevo ambiente (Devall, 2009). Las poblaciones de L. campestris y $L$. exaltatus, provenientes de mayor altitud, presentaron una supervivencia mayor que las de L. montanus, lo que puede atribuirse a que el traslado altitudinal fue menor en las primeras y están en niveles de humedad similar al sitio de prueba. Igualmente, las primeras pueden tener plasticidad fenotípica (McDowell et al., 2008; Mátyás et al., 2010). Este comportamiento es similar a lo reportado para L. elegans Kunth (Soto-Correa et al., 2015).

\section{Características agronómicas}

En la mayoría de las variables se detectaron diferencias significativas entre especies y poblaciones. En biomasa y longitud de vaina las diferencias fueron sólo entre poblaciones (Cuadro 2). El número de óvulos abortados y semillas dañadas totales no presentó diferencias entre 
Cuadro 2. Análisis de varianza en supervivencia y variables morfológicas de varias poblaciones de Lupinus campestris, $L$. exaltatus y L. montanus establecidas en un ensayo de campo en Tlachichuca, Puebla.

\begin{tabular}{|c|c|c|c|c|c|c|}
\hline \multirow{2}{*}{\multicolumn{2}{|c|}{ Variable }} & \multicolumn{2}{|c|}{ Significancia (Valor de P) } & \multicolumn{3}{|c|}{ Valores medios } \\
\hline & & Especies & Poblaciones & L. campestris & L. exaltatus & L. montanus \\
\hline \multicolumn{2}{|c|}{ Supervivencia (\%) } & 0.0001 & 0.0001 & $54.0 \mathrm{a}$ & $61.0 \mathrm{a}$ & $29.0 \mathrm{~b}$ \\
\hline \multicolumn{2}{|l|}{ Altura (cm) } & 0.0001 & 0.0001 & $100.9 b$ & $97.4 b$ & $136.3 \mathrm{a}$ \\
\hline \multicolumn{2}{|c|}{ Biomasa por planta (g) } & 0.2263 & 0.0031 & $212.1 \mathrm{a}$ & $208.0 \mathrm{a}$ & $205.2 \mathrm{a}$ \\
\hline \multicolumn{2}{|c|}{ Longitud de inflorescencia (cm) } & 0.0001 & 0.0001 & $12.6 b$ & $13.2 b$ & $20.4 \mathrm{a}$ \\
\hline \multirow{3}{*}{ Vainas } & Longitud (cm) & 0.0942 & 0.0001 & $3.9 \mathrm{a}$ & $3.96 a$ & $3.9 \mathrm{a}$ \\
\hline & Ancho (cm) & 0.0001 & 0.0001 & $0.6 b$ & $0.6 b$ & $0.7 \mathrm{a}$ \\
\hline & No. de semillas & 0.0001 & 0.0003 & $6.6 \mathrm{a}$ & $6.4 b$ & $6.2 \mathrm{c}$ \\
\hline \multirow{6}{*}{$\begin{array}{l}\text { Número } \\
\text { promedio } \\
\text { por planta }\end{array}$} & Vainas & 0.0001 & 0.0002 & $347.4 \mathrm{a}$ & $341.0 \mathrm{a}$ & $170.3 b$ \\
\hline & Óvulos abortados & 0.2635 & 0.0659 & $277.5 \mathrm{a}$ & $262.3 \mathrm{a}$ & $188.7 \mathrm{a}$ \\
\hline & Semillas Ilenas & 0.0003 & 0.0005 & 1006.0 a & $982.0 \mathrm{a}$ & $391.0 \mathrm{~b}$ \\
\hline & Semillas vanas & 0.0140 & 0.2219 & $461.0 \mathrm{a}$ & $546.0 \mathrm{a}$ & $241.0 \mathrm{~b}$ \\
\hline & Semillas dañadas & 0.2239 & 0.0791 & $40.0 \mathrm{a}$ & $41.0 \mathrm{a}$ & $19.0 \mathrm{a}$ \\
\hline & Semillas (g) & 0.0006 & 0.0841 & $14.7 \mathrm{a}$ & $13.8 \mathrm{a}$ & $6.1 \mathrm{~b}$ \\
\hline \multirow{3}{*}{ Semillas } & Ancho (mm) & 0.0001 & 0.0001 & $2.5 b$ & $2.5 b$ & $2.8 \mathrm{a}$ \\
\hline & Longitud (mm) & 0.0001 & 0.0001 & $3.5 b$ & $3.5 b$ & $3.7 \mathrm{a}$ \\
\hline & Peso 100 semillas (g) & 0.0001 & 0.0001 & $1.4 \mathrm{~b}$ & $1.4 \mathrm{~b}$ & $1.7 \mathrm{a}$ \\
\hline
\end{tabular}

Letras diferentes en hileras indican diferencias entre las especies de Lupinus $(P \leq 0.05)$.

especies y entre poblaciones (Cuadro 2). Las plantas de L. montanus presentaron el mayor valor en altura $(136 \mathrm{~cm}$ promedio) y L. exaltatus el menor $(98 \mathrm{~cm}$ ) (Cuadro 3), datos que son similares a los reportados por Lagunes-Espinoza et al. (2012) en poblaciones naturales de L. campestris con amplitudes entre 30 y $120 \mathrm{~cm}$, en L. exaltatus entre 30 y $110 \mathrm{~cm}$ y en L. montanus entre 70 y $240 \mathrm{~cm}$, así como por Zamora-Natera y Terrazas (2012), quienes refieren valores menores a $150 \mathrm{~cm}$ en L. montanus y de $190 \mathrm{~cm}$ en L. exaltatus, respectivamente. El rendimiento de biomasa ha $^{-1}$ con densidad de 1 semilla $\mathrm{m}^{-2}$ fue de $2.12 \mathrm{t} \mathrm{ha}^{-1}$ para L. campestris, de $2.08 \mathrm{t} \mathrm{ha}^{-1}$ para L. exaltatus y de $2.05 \mathrm{t}$ ha $^{-1}$ para L. montanus. Estos valores podrían aumentar si se cultivaran en densidades mayores y posiblemente alcanzarían rendimientos similares a lo reportado para L. albus L. (11.2 tha $\left.\mathrm{t}^{-1}\right)$ y L. mutabilis Sweet $\left(9.8 \mathrm{t} \mathrm{ha}^{-1}\right)$ en una densidad de 100 y 120 semillas $\mathrm{m}^{-2}$, respectivamente (Mikić et al., 2013). Otro factor que origina las diferencias en altura y peso en diferentes especies vegetales es la diferenciación genética y su interacción con el ambiente (López-Baez et al.; 2018; Rehfeldt, 2004; Viveros-Viveros et al., 2009).
Lupinus montanus presentó la mayor longitud de las inflorescencias y L. campestris la menor (Cuadro 4). La longitud de la inflorescencia totalmente desarrollada de las tres especies analizadas fue de 4 a $18.5 \mathrm{~cm}$, de 1.5 a 9.5 $\mathrm{cm}$ y de 11.6 a $45 \mathrm{~cm}$ para $L$. campestris, $L$. exaltatus y $L$. montanus respectivamente, similar a la reportada para las mismas especies en poblaciones naturales por LagunesEspinoza et al. (2012). Las flores de Lupinus forman racimos al final de la rama, y de acuerdo con Walker (2011), su formación tarda de 4 a 8 semanas aproximadamente. Para las especies analizadas tal duración fue de 4 a 5 semanas en promedio.

Walker y Luckett (2011) mencionaron que la formación de flores inicia una vez que se detiene el crecimiento del tallo y de las hojas, y el tamaño final de la inflorescencia depende de la especie, fase vegetativa de la planta y del tamaño de la planta en particular; además, la floración depende del número de horas frío acumuladas por la planta y la respuesta de la planta varía con la especie de Lupinus e incusive con la variedad. La falta de horas frío permite crecimiento continuo de hojas, sin floración, o genera flores anormales, con pétalos estrechos. Así, la 
Cuadro 3. Supervivencia y variables morfológicas de varias poblaciones de L. campestris, L. exaltatus y L. montanus establecidas en un ensayo de campo en Tlachichuca, Puebla.

\begin{tabular}{|c|c|c|c|c|c|c|c|c|c|}
\hline \multirow[b]{2}{*}{ Especie/Población } & \multirow[b]{2}{*}{ Supervivencia (\%) } & \multicolumn{3}{|c|}{ Promedio por planta } & \multicolumn{5}{|c|}{ Número promedio por planta } \\
\hline & & Altura $(\mathrm{cm})$ & Biomasa (g) & Semilla (g) & Vainas & $\begin{array}{c}\text { Óvulos } \\
\text { abortados }\end{array}$ & $\begin{array}{l}\text { Semillas } \\
\text { Ilenas }\end{array}$ & $\begin{array}{c}\text { Semillas } \\
\text { vanas }\end{array}$ & $\begin{array}{l}\text { Semillas } \\
\text { dañadas }\end{array}$ \\
\hline \multicolumn{10}{|l|}{ L. campestris } \\
\hline Barranca Honda & $50.0 \mathrm{ab}$ & $122.0 \mathrm{c}$ & $267.3 \mathrm{a}$ & $16.1 \mathrm{ab}$ & $269 \mathrm{bc}$ & 158 a & 1038 bc & $424 a$ & $41 \mathrm{a}$ \\
\hline Laguna de Atexca & $52.7 \mathrm{ab}$ & 95.0 de & $196.5 b$ & $15.8 a b$ & $401 \mathrm{~b}$ & $381 \mathrm{a}$ & 1073 bc & $534 a$ & $16 a$ \\
\hline Laguna Seca & $47.2 \mathrm{ab}$ & $109.7 \mathrm{~cd}$ & $257.5 \mathrm{a}$ & $16.0 \mathrm{ab}$ & $371 \mathrm{bc}$ & $341 \mathrm{a}$ & 1037 bc & $467 \mathrm{a}$ & $125 \mathrm{a}$ \\
\hline Poxcoatzingo & $36.1 \mathrm{~b}$ & $72.5 \mathrm{e}$ & $195.3 b$ & $5.1 \mathrm{ab}$ & $222 \mathrm{~cd}$ & $281 \mathrm{a}$ & $361 c$ & $272 a$ & $10 a$ \\
\hline San Isidro & $47.2 \mathrm{ab}$ & 100.6 de & $183.0 \mathrm{bc}$ & $12.4 a b$ & $283 \mathrm{bc}$ & $214 a$ & 875 bc & $250 \mathrm{a}$ & $8 a$ \\
\hline Tlalmotolo I & $63.8 \mathrm{a}$ & 96.6 de & $236.0 a b$ & $27.6 \mathrm{a}$ & $604 \mathrm{a}$ & $207 \mathrm{a}$ & 2050 a & $635 a$ & $33 a$ \\
\hline Tlalmotolo II & $66.6 \mathrm{a}$ & $102.1 \mathrm{de}$ & $158.0 \mathrm{bc}$ & $16.2 a b$ & 358 bc & $263 a$ & 1050 bc & $508 a$ & $73 a$ \\
\hline Zoapan & $61.1 \mathrm{a}$ & $105.0 \mathrm{~d}$ & $207.7 a b$ & $7.9 a b$ & $271 \mathrm{bc}$ & $375 a$ & $565 c$ & $603 a$ & $17 a$ \\
\hline \multicolumn{10}{|l|}{ L. exaltatus } \\
\hline Manuel Ávalos & $66.6 \mathrm{a}$ & 92.4 de & $162.5 \mathrm{bc}$ & $12.2 a b$ & 329 bc & $194 a$ & 871 bc & $558 \mathrm{a}$ & $19 a$ \\
\hline San Martín & $58.3 \mathrm{a}$ & 93.9 de & $232.9 a b$ & $17.5 \mathrm{ab}$ & $345 \mathrm{bc}$ & $235 a$ & $1210 b$ & $455 \mathrm{a}$ & $27 a$ \\
\hline Zoapan & $55.5 \mathrm{a}$ & $107.4 \mathrm{~cd}$ & $231.0 \mathrm{ab}$ & $12.3 a b$ & $349 \mathrm{bc}$ & $358 a$ & 866 bc & $626 a$ & $78 a$ \\
\hline \multicolumn{10}{|l|}{ L. montanus } \\
\hline Texmalaquilla 3100 & $36.1 \mathrm{~b}$ & $145.1 \mathrm{~b}$ & $213.8 a b$ & $0.3 b$ & $19 d$ & 32 a & $21 d$ & $28 a$ & $9 a$ \\
\hline Texmalaquilla 3300 & 19.4 C & $149.6 \mathrm{ab}$ & $245.0 \mathrm{ab}$ & $5.4 \mathrm{ab}$ & $111 \mathrm{~cd}$ & $106 a$ & $315 c$ & $141 \mathrm{a}$ & $32 a$ \\
\hline Texmalaquilla 3500 & $16.6 \mathrm{c}$ & $137.3 \mathrm{bc}$ & $183.1 \mathrm{abc}$ & $6.1 \mathrm{ab}$ & $119 \mathrm{~cd}$ & 89 a & $413 c$ & $22 \mathrm{a}$ & $2 a$ \\
\hline Texmalaquilla3700 & $25.0 \mathrm{bc}$ & $125.4 \mathrm{bcd}$ & $164.0 \mathrm{bc}$ & $12.7 \mathrm{ab}$ & $288 \mathrm{bcd}$ & $224 a$ & 779 bc & $272 a$ & $4 a$ \\
\hline Texmalaquilla 3900 & 19.4 bc & 174.6 a & $267.6 \mathrm{a}$ & $5.8 \mathrm{ab}$ & $149 \mathrm{~cd}$ & 64 a & $345 c$ & $209 a$ & $58 a$ \\
\hline Texmalaquilla 4100 & $33.3 \mathrm{bc}$ & $126.8 \mathrm{bc}$ & $262.8 \mathrm{ab}$ & $4.9 \mathrm{ab}$ & 300 bc & $653 a$ & $303 c$ & $706 a$ & $8 a$ \\
\hline San Joaquin & $36.1 \mathrm{~b}$ & $105.5 \mathrm{~cd}$ & 150.4 bc & $8.7 \mathrm{ab}$ & $255 c$ & 328 a & $609 c$ & $469 a$ & $45 a$ \\
\hline Tlanalapan & 38.8 bc & $122.7 \mathrm{bcd}$ & $237.6 a b$ & $6.4 a b$ & $195 \mathrm{~cd}$ & $62 \mathrm{a}$ & $492 \mathrm{c}$ & $171 \mathrm{a}$ & $13 a$ \\
\hline Zoapan & $36.1 \mathrm{~b}$ & 139.8 bc & $122.6 \mathrm{c}$ & $3.5 b$ & $97 \mathrm{~cd}$ & $139 a$ & $245 c$ & $151 \mathrm{a}$ & $7 \mathrm{a}$ \\
\hline
\end{tabular}

Letras diferentes en columnas indican diferencias entre las poblaciones de Lupinus $(P \leq 0.05)$.

temperatura puede llegar a retrasar la fenología de las especies y originar daño en fechas posteriores por heladas repentinas (Reader et al., 1995; Walker y Luckett, 2011).

El factor temperatura puede ser crucial para L. montanus, especie en la cual no se desarrolló la inflorescencia de forma adecuada. Las nueve poblaciones evaluadas de esta especie fueron trasladadas de sus lugares de origen a un sitio de menor elevación, mayor temperatura promedio y menor precipitación (Cuadro 1); por lo tanto, estuvo sujeta a un menor número de horas frío y humedad insuficiente para la iniciación floral y formación de estructuras reproductivas.

Lupinus campestris fue la especie que presentó la mayor producción de vainas (347 vainas/planta) y $L$. montanus la menor (170 vainas/planta); así mismo, entre las poblaciones se presentó variabilidad (Cuadro 4), lo que sugiere diferencias entre genotipos moldeados por la interacción ambiental, donde la temperatura y humedad juegan un papel crucial (Dracup y Kirby, 1996; Pospišil y Pospišil, 2015). En el caso de L. campestris, Águila et al. (2018) reportaron para la población de Tlalmotolo valores menores (290 vainas), mientras que en el presente estudio los valores fueron de 358 a 604 vainas para Tlalmotolo II y Tlalmotolo I, respectivamente, en un ambiente donde la temperatura promedio es menor.

Se menciona que la producción de vainas depende de una polinización adecuada (Parker y Edwards, 2011). De cualquier forma, estas especies silvestres presentan una productividad mayor en comparación con especies cultivadas o domesticadas; por ejemplo, las variedades Fedora y Energy de L. albus, así como la variedad Arabella de $L$. angustifolius presentaron de 4 a 9 vainas/planta (Pospišil y Pospišil, 2015), aunque en trabajos con L. albus que crecieron en diferentes ambientes se han reportado valores desde 9 hasta 45 vainas/plantas (Annicchiarico et 
al., 2010; González-Andrés et al., 2007; Teferi et al., 2015).

Las tres especies presentaron una longitud de vaina similar, de $3.90 \mathrm{~cm}$ en promedio. L. montanus presentó $10 \%$ más anchura de vainas que las otras dos especies (Cuadro 4), valor semejante a lo reportado por LagunesEspinoza et al. (2012) en poblaciones naturales.

El número de semillas por vaina fue similar para $L$. campestris y $L$. exaltatus (seis semillas); mientras que L. montanus presentó valores de cinco y seis semillas. La variación entre poblaciones puede ser efecto de las condiciones ambientales en el momento de la formación de la estructura floral y la polinización (Walker, 2011).
El número de óvulos abortados fue mayor para L. campestris, con un promedio de 277, valor mayor al de poblaciones naturales de los mismos sitios (Águila et al., 2018). En L. montanus esta variable mostró el menor valor, con 188. En semillas llenas por planta L. campestris tuvo el valor mayor con 1006 mientras que L. montanus fue la especie con menos semillas por planta. Los mayores valores promedio en semillas vanas fueron para $L$. exaltatus, en tanto que L. montanus presentó los valores más bajos (Cuadro 3). L. montanus exhibió el promedio menor de semillas dañadas por insectos por planta y $L$. campestris el valor mayor (Cuadro 3).

Cuadro 4. Características de inflorescencia y semillas de Lupinus campestris, L. exaltatus y L. montanus establecidos en un ensayo de campo en Tlachichuca, Puebla.

\begin{tabular}{|c|c|c|c|c|c|c|c|}
\hline \multirow[b]{2}{*}{ Especie/Población } & \multicolumn{4}{|c|}{ Inflorescencia } & \multicolumn{3}{|c|}{ Semilla } \\
\hline & $\begin{array}{l}\text { Longitud } \\
\quad(\mathrm{cm})\end{array}$ & $\begin{array}{l}\text { Longitud de } \\
\text { vaina }(\mathrm{cm})\end{array}$ & $\begin{array}{l}\text { Ancho de } \\
\text { vaina }(\mathrm{cm})\end{array}$ & $\begin{array}{l}\text { Semillas } \\
\text { por vaina }\end{array}$ & Ancho (mm) & $\begin{array}{l}\text { Longitud } \\
(\mathrm{mm})\end{array}$ & $\begin{array}{c}\text { Peso } 100 \\
\text { semillas } \\
(\mathrm{g})\end{array}$ \\
\hline \multicolumn{8}{|l|}{ L. campestris } \\
\hline Barranca Honda & $10.6 f$ & $4.0 \mathrm{ab}$ & $0.6 \mathrm{e}$ & $6 \mathrm{bc}$ & $2.7 \mathrm{bc}$ & $3.6 \mathrm{~cd}$ & $1.5 \mathrm{a}$ \\
\hline Laguna de Atexca & $13.5 \mathrm{e}$ & $3.8 \mathrm{c}$ & $0.6 \mathrm{de}$ & $6 b$ & $2.4 \mathrm{~d}$ & 3.4 de & $1.4 \mathrm{~cd}$ \\
\hline Laguna Seca & $12.9 \mathrm{e}$ & $3.9 \mathrm{bc}$ & 0.6 de & $6 \mathrm{bc}$ & $2.6 \mathrm{~cd}$ & $3.5 \mathrm{~cd}$ & $1.3 \mathrm{~cd}$ \\
\hline Manuel Ávalos & $12.8 \mathrm{e}$ & $3.9 \mathrm{bc}$ & $0.6 \mathrm{e}$ & $6 \mathrm{bc}$ & $2.6 \mathrm{~cd}$ & $3.6 \mathrm{~cd}$ & $1.4 \mathrm{~cd}$ \\
\hline Tlalmotolo | & $13.5 \mathrm{e}$ & $3.7 d$ & 0.6 de & $6 \mathrm{bc}$ & $2.4 \mathrm{~d}$ & $3.4 \mathrm{e}$ & $1.3 \mathrm{~cd}$ \\
\hline Tlalmotolo II & $13.8 \mathrm{e}$ & $4.0 \mathrm{~b}$ & $0.6 c$ & $6 b$ & $2.6 c$ & $3.6 \mathrm{~cd}$ & $1.6 \mathrm{~b}$ \\
\hline Poxcoatzingo & $12.0 \mathrm{ef}$ & $3.7 \mathrm{~cd}$ & $0.6 \mathrm{~cd}$ & $6 \mathrm{bc}$ & $2.5 \mathrm{~cd}$ & $3.6 \mathrm{~cd}$ & $1.4 \mathrm{~cd}$ \\
\hline Zoapan & $12.1 \mathrm{ef}$ & $3.9 \mathrm{bc}$ & $0.6 \mathrm{~cd}$ & $7 a$ & $2.6 \mathrm{c}$ & $3.7 \mathrm{c}$ & $1.4 \mathrm{c}$ \\
\hline \multicolumn{8}{|l|}{ L. exaltatus } \\
\hline San Isidro & $13.6 \mathrm{e}$ & $3.9 \mathrm{bc}$ & $0.6 \mathrm{de}$ & $6 \mathrm{bc}$ & $2.5 \mathrm{~cd}$ & $3.5 d$ & $1.4 \mathrm{~cd}$ \\
\hline San Martín & $12.6 \mathrm{e}$ & $3.9 \mathrm{bc}$ & 0.6 de & $6 b$ & $2.4 d$ & $3.4 \mathrm{de}$ & $1.1 \mathrm{~d}$ \\
\hline Zoapan & $13.5 \mathrm{e}$ & $3.9 \mathrm{bc}$ & $0.6 d$ & $6 a$ & $2.6 \mathrm{c}$ & $3.6 \mathrm{~cd}$ & $1.4 \mathrm{c}$ \\
\hline \multicolumn{8}{|l|}{ L. montanus } \\
\hline San Joaquín & $12.8 \mathrm{e}$ & $3.8 \mathrm{~cd}$ & $0.6 \mathrm{~cd}$ & $6 \mathrm{bc}$ & $2.5 \mathrm{~cd}$ & $3.7 \mathrm{c}$ & $1.6 b$ \\
\hline Texmalaquilla 3100 & $20.5 c$ & $4.2 \mathrm{a}$ & $0.7 \mathrm{a}$ & $6 \mathrm{bc}$ & $3.1 \mathrm{a}$ & $4.2 \mathrm{a}$ & $1.7 \mathrm{ab}$ \\
\hline Texmalaquilla 3300 & $28.6 \mathrm{a}$ & $3.8 \mathrm{bcd}$ & $0.7 \mathrm{ab}$ & $6 \mathrm{bc}$ & $3.0 \mathrm{ab}$ & $3.7 \mathrm{~cd}$ & $1.7 \mathrm{ab}$ \\
\hline Texmalaquilla 3500 & $16.5 \mathrm{~cd}$ & $3.7 \mathrm{~cd}$ & 0.6 cde & $6 a b c$ & $3.0 \mathrm{ab}$ & $3.7 \mathrm{c}$ & $1.1 \mathrm{ab}$ \\
\hline Texmalaquilla 3700 & $20.8 \mathrm{bc}$ & $3.8 \mathrm{bcd}$ & $0.6 \mathrm{~cd}$ & $5 c$ & $2.5 \mathrm{~cd}$ & $3.7 \mathrm{c}$ & $1.6 \mathrm{bc}$ \\
\hline Texmalaquilla 3900 & $18.9 \mathrm{~cd}$ & $4.0 \mathrm{ab}$ & $0.7 a b$ & $7 a$ & $2.5 \mathrm{~cd}$ & $3.6 \mathrm{~cd}$ & $1.6 \mathrm{bc}$ \\
\hline Texmalaquilla 4100 & $24.0 \mathrm{~b}$ & $3.8 \mathrm{bcd}$ & $0.7 a b$ & $6 c$ & $2.8 b$ & $3.7 \mathrm{c}$ & $1.8 a b$ \\
\hline Tlanalapan & $22.4 \mathrm{bc}$ & $3.7 \mathrm{~cd}$ & $0.7 \mathrm{bc}$ & $6 \mathrm{bc}$ & $2.6 \mathrm{bcd}$ & $3.5 \mathrm{~cd}$ & $1.6 \mathrm{abc}$ \\
\hline Zoapan & $18.9 \mathrm{~cd}$ & $3.9 \mathrm{bc}$ & $0.7 \mathrm{~b}$ & $6 \mathrm{bc}$ & $2.9 a b$ & $4.0 \mathrm{~b}$ & $1.8 \mathrm{a}$ \\
\hline
\end{tabular}

Letras diferentes en columnas indican diferencias entre las poblaciones de Lupinus $(P \leq 0.05)$. 
Los valores observados en óvulos abortados pueden deberse a la falta de polen derivado por la asincronia de las estructuras reproductivas, falta de polen y baja disponibilidad de insectos polinizadores, todo ello indispensable para la polinización cruzada (Dracup et al., 1998; Farrington y Pate, 1981). En estudios con L. angustifolius y $L$. albus se ha determinado que el ambiente es más importante en la sincronización floral que el componente genético (Walker y Luckett, 2011). Ma et al. (1998) señalaron que el porcentaje de semillas dañadas y vanas es producto de efectos ambientales, principalmente por temperatura y aspectos nutrimentales o por el ataque de insectos, hongos o áfidos (Hertel et al., 2013).

Con respecto a los caracteres de la semilla, L. montanus presentó, en promedio, el mayor ancho y $L$. exaltatus el menor (Cuadro 4). El mayor largo de la semilla fue para $L$. montanus, con un promedio de $3.79 \mathrm{~mm}$. Pablo-Pérez et al. (2013) reportó valores menores para la misma especie. El intervalo para el peso de 100 semillas en las poblaciones de las tres especies fue entre 1.36 y $1.84 \mathrm{~g}$. Las semillas más pesadas las produjo $L$. montanus con un valor promedio de $1.70 \mathrm{~g}$, mientras que las más ligeras fueron las de $L$. exaltatus con $1.41 \mathrm{~g}$ por 100 semillas. En general estas semillas resultaron menos pesadas en comparación con las producidas in situ, de acuerdo con Lagunes-Espinoza et al. (2012), en las mismas poblaciones muestreadas que para este experimento. Lo anterior sugiere que el mover estas especies de Lupinus a sitios con diferentes condiciones ambientales influye sobre la productividad de la semilla. Para la producción de semilla por hectárea con densidad de 1 semilla $\mathrm{m}^{-2}$, y considerando el promedio por planta (Cuadro 2), se estima que podría ser de 146.4, 138.6 y $61.3 \mathrm{~kg} \mathrm{ha}^{-1}$ en L. campestris, L. exaltatus y L. montanus, respectivamente.

En cada especie se detectó diversidad significativa entre las poblaciones probadas; sin embargo, para este tipo de evuaciones es importante considerar la procedencia de las poblaciones, pues la temperatura y humedad son importantes para cada una de las especies, en particular para obtener alto porcentaje de supervivencia y de producción en campo. Es necesario establecer ensayos que permitan delimitar el movimiento para las tres especies, las cuales, al parecer muestran escasa plasticidad fenotípica, lo que tendría implicaciones agronómicas y ecológicas.

\section{CONCLUSIONES}

La variación en la superviencia, altura de planta, número de semillas y peso de semillas fue significativa entre especies y entre poblaciones de Lupinus. En los caracteres de semillas, biomasa y número de vaina $L$. campestris y $L$. exaltatus presentaron mejor desempeño que $L$. montanus, características que las hacen promisorias para su domesticación bajo las condiciones ambientales evaluadas. La supervivencia y bajo rendimiento de semillas de L. montanus, en comparación con las otras especies evaluadas, es resultado del establecimiento del ensayo a una altitud menor a su distribución natural y refleja falta de plasticidad fenotípica al ambiente.

\section{AGRADECIMIENTOS}

Al Colegio de Postgraduados por el apoyo en el financiamiento del proyecto.

\section{BIBLIOGRAFÍA}

Águila S. I., O. Vázquez C., J. López U., A. López L., E. Martínez R., E. García G y E. M. Zamora C. (2018) Variación morfológica y reproductiva de nueve poblaciones naturales de Lupinus campestris Cham. \& Schltdl. de la región centro oriente de Puebla, México. Revista Biológico Agropecuaria Tuxpan 6(Núm Esp.):2137-2142.

Allen R. G., L. S. Pereira, D. Raes and M. Smith (1998) Crop Evapotranspiration (Guidelines for Computing Crop Water Requirements). FAO Irrigation and Drainage Paper No.56. FAO, Water Resources, Development and Management Service. Rome, Italy. 300 p.

Annicchiarico P., N. Harzic and A. M. Carroni (2010) Adaptation, diversity, and exploitation of global white lupin (Lupinus albus L.) landrace genetic resources. Field Crops Research 119:114-124 https://doi.org/10.1016/j.fcr.2010.06.022

Bermúdez-Torres K., J. Martínez H., R. Figueroa B., M. Wink and L. Legal (2009) Activity of quinolizidine alkaloids from three Mexican Lupinus against the lepidopteran crop pest Spodoptera frugiperda. BioControl 54:459-466, https://doi.org/10.1007/ s10526-008-9180-y

Chen Y. L., V. M. Dunbabin, J. A. Postma, A. J. Diggle, J. A. Palta, J. P. Lynch, K. H. M. Siddique and Z. Rengel (2011) Phenotypic variability and modelling of root structure of wild Lupinus angustifolius genotypes. Plant and Soil 348:345, https://doi.org/10.1007/ s11104-011-0939-z

Devall M. S. (2009) Efectos del cambio climático mundial en los árboles y arbustos raros. Unasylva 60:231-232.

Dracup M. and E. J. M. Kirby (1996) Pod and seed growth and development of narrow-leafed lupin in water limited Mediterranean-type environment. Field Crops Research 48:200-222, https://doi org/10.1016/S0378-4290(96)00040-8

Dracup M., B. Thomson, M. Reader, E. J. M. Kirby, I. Shield and J. Leach (1998) Daylength responses, flowering time and seed filling in lupins. Australian Journal of Agricultural Research 49:1047-1056, https://doi.org/10.1071/A98033

Farrington P. and J. S. Pate (1981) Fruit set in Lupinus angustifolius cv. Unicrop. I. Phenology and growth during flowering and early fruiting. Australian Journal of Plant Physiology 8:293-305 https://doi.org/10.1071/PP9810293

González-Andrés F., P. A. Casquero, C. San-Pedro and E. Hernández-Sánchez (2007) Diversity in white lupin (Lupinus albus L.) landraces of Northwest Iberian plateau. Genetic Resources and Crop Evolution 54:27-44, https://doi.org/10.1007/s10722-0051407-5

Hernández F. E., R. K. Rivera M., O. J. Ramos H., F. C. Salinas P., M. Rodríguez M. and K. Bermúdez T. (2008) Effect of scarification treatments on germination of Lupinus montanus HBK seeds. In: Lupins for Health and Wealth. J. A. Palta and J. B. Berger (eds.). Lupins for Health and Wealth, Proceedings of the 12th International Lupin Conference. Fremantle, Western Australia. International Lupin Association. Canterbury, New Zealand. pp:405-409.

Hertel K., K. Roberts and P. Bowden (2013) Insect and Mite Control in Field Crops. NSW Department of Primary Industry. Orange, Australia. $103 p$.

Lagunes-Espinoza L. C., C. Huyghe and J. Papineau (2000) Genetic variation 
for pod wall proportion in Lupinus albus. Plant Breeding 1 19:421425, https://doi.org/10.1046/j.1439-0523.2000.00525.x

Lagunes-Espinoza L. C., J. López-Upton, E. García-López, J. Jasso-Mata, A. Delgado-Alvarado y G. García S. (2012) Diversidad morfológica y concentración de proteína de Lupinus spp. en la región centrooriental del estado de Puebla, México. Acta Botánica Mexicana 99:73-90.

Lagunes-Espinoza L. C., M. Pablo-Pérez, E. M. Aranda-Ibáñez, J. López-Upton y J. Ramos-Juárez (2013) Potencial nutritivo para alimentación animal de leguminosas silvestres del género Lupinus del estado de Puebla. In: XII Simposio Internacional y VII Congreso Nacional de Agricultura Sostenible. F. Álvares G., F. Bahena J., I. Carranza C., R. Díaz R., I. Ocampo. F, E. Ortíz T., A. Pérez M., E. Pérez R., J. A. Villanueva J. y L. A. Villareal M. (eds.). Sociedad Mexicana de Agricultura Sostenible. Puebla, México. pp:29792988.

Lara-Cabrera S. I., N. Alejandre-Melena, E. I. Medina-Sánchez and R. LindigCisneros (2009) Genetic diversity in populations of Lupinus elegans Kunth, implications for ecological restoration. Revista Fitotecnia Mexicana 32:79-86.

Littell R. C., G. A. Milliken, W. W. Stroup, R. D. Wolfinger and O. Schabenberger (1996) SAS System for Mixed Models. Second edition. Statistical Analysis System Institute. Cary, NC. 633 p.

López-Baez L. I., O. R. Taboada-Gaytán, A. Gil-Muñoz, P. A. López, E. OrtizTorres, M. L. P. Vargas-Vázquez y R. Díaz-Cervantes (2018) Diversidad morfoagronómica del frijol ayocote en el Altiplano CentroOriente de Puebla. Revista Fitotecnia Mexicana 47:487-497.

López-Bellido L., M. Fuentes, J. C. B. Lhamby and J. E. Castillo (1994) Grow and yield of white lupin (Lupinus albus) under Mediterranean conditions: effect of sowing date. Field Crops Research 36:8794, https://doi.org/10.1016/0378-4290(94)90057-4

López-Upton J., C. Ramírez-Herrera, 0. Plascencia-Escalante y J. Jasso-Mata (2004) Variación en crecimiento de diferentes poblaciones de las dos variedades de Pinus greggii. Agrociencia 38:457-464.

Ma Q., N. Longnecker, N. Emery and C. Atkins (1998) Growth and yield in Lupinus angustifolius are depressed by early transient nitrogen deficiency. Australian Journal of Agricultural Research 49:811820, https://doi.org/10.1071/A97153

Mátyás C., I. Berki, B. Czúcz, B. Gálos, N. Móricz and E. Rasztovits (2010) Future of beech in Southeast Europe from the perspective of evolutionary ecology. Acta Silvatica et Lignaria Hungarica 6:91110.

McDowell N., W. T. Pockman, C. D. Allen, D. D. Breshears, N. Cobb, T. Kolb, J. Plaut, J. Sperry, A. West, D. G. Williams and E. A. Yepez (2008) Mechanisms of plant survival and mortality during drought: why do some plants survive while others succumb to drought? New Phytologist 178:719-739, https://doi.org/10.1111/j.14698137.2008.02436.x

Mikić A., B. Ćupina, V. Mihailović, Đ. Krstić, S. Antanasović, L. Zorić, V. Đordevic, V. Perić and M. Srebrić (2013) Intercropping white (Lupinus albus) and Andean (Lupinus mutabilis) lupins with other annual cool season legumes for forage production. South African Journal of Botany 89:296-300, https://doi.org/10.1016/j.sajb.2013.06.015

Pablo-Pérez M., L. C. Lagunes-Espinoza, J. López-Upton, J. Ramos-Juárez y E. M. Aranda-lbáñez (2013) Morfometría, germinación, y composición mineral de semillas de Lupinus silvestres. Bioagro 25:101-108

Pablo-Pérez M., L. C. Lagunes-Espinoza, J. López-Upton, E. M. Aranda-Ibáñez y J. Ramos-Juárez (2015) Composición química de especies silvestres del género Lupinus del estado de Puebla, México. Revista Fitotecnia Mexicana 38:49-55.

Parker P. and J. Edwards (2011) Pod and seed development In: Lupin Growth and Development. J. Edwards, J. Walker and G. McIntosh (eds.). NSW Department of Industry and Investment. Sidney, Australia. pp:63-79.
Pospišil A. and M. Pospišil (2015) Influence of sowing density on agronomic traits of lupins (Lupinus spp.). Plant, Soil and Environment 9:422-425, https:// doi.org/10.17221/436/2015-PSE

Quinn G. P. and M. J. Keough (2002) Experimental Design and Data Analysis for Biologists. Cambridge University Press. New York. $537 \mathrm{p}$.

Reader M. A., M. Dracup and E. J. M. Kirby (1995) Time to flowering in narrow-leafed lupin. Australian Journal of Agricultural Research 46:1063-1077, https://doi.org/10.1071/AR9951063

Rehfeldt G. E. (2004) Interspecific and intraspecific variation in Picea engelmanii and its congeneric cohorts: biosystematics, genecology and climate change. General Technical Report RMRS-GTR-134. USDA Forest Service. Fort Collins, Colorado. 18 p, https://doi.org/10.2737/RMRS-GTR-134

Ruiz-López M. A., P. M. García-López, H. Castañeda-Vázquez, N. J. F. Zamora, P. Garzón-De la Mora, J. Bañuelos P., C. Burbano, M. M. Pedrosa, C. Cuadrado and M. Muzquiz (2000) Chemical composition and antinutrient content of three Lupinus species from Jalisco, México. Journal of Food Composition and Analysis 13:193-199. https://doi.org/10.1006/jfca.1999.0887

Sáenz-Romero C., G. E. Rehfeldt, N. L. Crookston, P. Duval, R. StAmant, J. Beaulieu and B. A. Richardson (2010) Spline models of contemporary, 2030, 2060 and 2090 climates for Mexico and their use in understanding climate-change impacts on the vegetation. Climatic Change 102:595-623 https://doi org/10.1007/s10584-009-9753-5

SAS Institute (2004) SAS/STAT® 9.1 User's Guide. SAS Institute Inc. Cary, NC, USA. $4420 \mathrm{p}$.

Soto-Correa J. C., C. Sáenz-Romero, R. Lindig-Cisneros, N. Sánchez-Vargas y J. Cruz-de-León (2012) Variación genética entre procedencias de Lupinus elegans Kunth, zonificación altitudinal y migración asistida. Agrociencia 46:593-608

Soto-Correa J. C., C. Sáenz-Romero, H. Paz and R. Lindig-Cisneros (2015) Drought stress in provenances of Lupinus elegans from different altitudes. Madera y Bosques 21:35-43.

Teferi T. A., M. Legesse and T. Birhane (2015) Searching and testing of white lupine (Lupinus albus L.) for adaptation and resistant to crenate broomrape in Tigray, Ethiopia. World Journal of Agriculture Sciences 11:341-345

Vega-Villasante F., H. Nolasco, C. Montaño, H. Romero-Schmidt y E. VegaVillasante (1996) Efecto de la temperatura, acidez, iluminación, salinidad, irradiación solar y humedad sobre la germinación de semillas de Pachycereus pecten-aboriginum "cardón barbón" (Cactaceae). Cactáceas y Suculentas Mexicanas 41:51-61.

Viveros-Viveros H., C. Sáenz-Romero, J. J. Vargas-Hernández, J. López-Upton, G. Ramírez-Valverde and A. Santacruz-Varela (2009) Altitudinal genetic variation in Pinus hartwegii Lindl. I: height growth, shoot phenology, and frost damage in seedlings. Forest Ecology and Management 257:836-842. https://doi.org/10.1016/j. foreco.2008.10.021

Walker J. (2011) Introduction. In: Lupin Growth y Development. J. Edwards, J. Walker and G. McIntosh (eds.). NSW Department of Industry and Investment. Sidney, Australia. pp:1-10.

Walker J. and D. Luckett (2011) Reproductive development. In: Lupin Growth and Development. J. Edwards, J. Walker and G McIntosh (eds.). NSW Department of Industry and Investment. Sidney, Australia. pp:47-62

Wink M. (1992) The role of quinolizidine alkaloids in plant-insect interactions. In: Insect-Plants Interactions. vol. IV. E. A. Bernays. (ed.). CRC Press. Boca Raton, FL, USA. pp:131-166.

Zamora-Natera J. F. y T. Terrazas (2012) Anatomía foliar y del pecíolo de cuatro especies de Lupinus (Fabaceae). Revista Mexicana de Biodiversidad 83:687-697. 